\title{
RTEL1, une \\ hélicase de I'ADN \\ essentielle \\ à la stabilité \\ du génome
}

> Le gène qui code l'hélicase de I'ADN RTELL (regulator of telomere elongation helicase 1) a été identifié chez l'homme il y a plus de 10 ans dans une région génétique associée au cancer gastrique. Depuis, plusieurs travaux, réalisés essentiellement chez le nématode Caenorhabditis elegans et la souris, ont mis à jour certaines caractéristiques fonctionnelles de RTELl et démontré son implication dans la stabilité chromosomique, le maintien de la longueur des télomères et la réparation de certaines lésions de I'ADN. De plus, récemment, quatre laboratoires ont identifié des mutations du gène codant RTELl chez des patients atteints de dyskératose congénitale et de sa forme sévère, le syndrome de Hoyeraal-Hreidarsson, ce qui démontre le rôle essentiel de RTELl dans l'homéostasie des télomères chez l'homme. Cependant, les fonctions précises de RTELl restent à ce jour mal définies. Dans cette synthèse, nous récapitulons les données concernant RTELl et discutons des autres activités que pourrait exercer ce facteur essentiel au maintien du génome. <

Le maintien de l'intégrité du génome est essentiel pour la survie cellulaire et la propagation de l'information génétique. Cependant, le génome est continuellement soumis à des agressions provenant de facteurs exogènes tels que les radiations ionisantes, les ultraviolets, ou des agents chimiques génotoxiques. Des lésions de l'ADN peuvent également survenir à la suite de certains processus biologiques endogènes, tels que la synthèse d'ATP dans les mitochondries (productrice de radicaux libres) ou la réplication. Les extrémités des chromosomes, les télomères, du fait de leurs séquences répétées riches en guanidine, représentent des régions particulièrement complexes à répliquer et sensibles aux attaques des agents pro-oxydants $[1,2]$. La réplication des extrémités des chromosomes eucaryotes est incomplète (end replication problem) et conduit à un raccourcissement

Tangui Le Guen ${ }^{1,2}$, Laurent Jullien ${ }^{1,2}$, Mike Schertzer ${ }^{4}$, Axelle Lefebvre, ${ }^{1,2}$, Laetitia Kermasson ${ }^{1,2}$, Jean-Pierre de Villartay ${ }^{1,2,3}$, Arturo Londoño-Vallejo ${ }^{4}$, Patrick Revy ${ }^{1,2}$

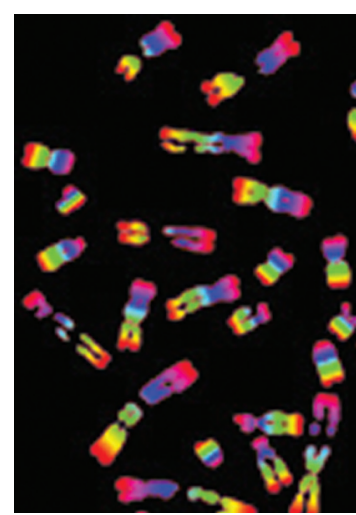

progressif de la taille des télomères après chaque division cellulaire [3]. La télomérase, un complexe ribonucléoprotéique exprimé spécifiquement dans
${ }^{1}$ Dynamique du génome et système immunitaire, Institut Imagine, 149, rue de Sèvres, 75015 Paris, France ;

${ }^{2}$ Inserm U768, université Paris Descartes, Sorbonne Paris Cité, Institut Imagine, 149, rue de Sèvres, 75015 Paris, France ; ${ }^{3}$ Hôpital Necker-Enfants malades, AP-HP, service d'immunologie et d'hématologie pédiatriques, 149, rue de Sèvres, 75015 Paris, France ;

${ }^{4}$ Télomère et cancer, Institut Curie, UMR3244, 26 Rue d'Ulm, 75005 Paris, France.

patrick.revy@inserm.fr les cellules germinales, les cellules souches et certaines cellules activées, compense cette perte [3]. Dans les autres cellules, l'érosion progressive des télomères conduit, à terme, à un arrêt de la prolifération : sénescence réplicative ou apoptose selon le contexte génétique $[4,5]$.

Une mauvaise prise en charge des dommages de l'ADN et/ou un mauvais maintien de l'intégrité des télomères provoquent chez l'homme des pathologies associées à une forte instabilité génétique et au développement de cancers. C'est pourquoi une multitude de facteurs protéiques contrôlent le bon déroulement des mécanismes impliqués dans le métabolisme de l'ADN (réplication, recombinaison, réparation, etc.) afin de garantir la stabilité du génome. Certains de ces facteurs, spécifiquement localisés aux télomères, participent à leur homéostasie et leur protection; d'autres sont nécessaires à la stabilité globale du génome. Plusieurs travaux récents ont permis d'identifier RTELl comme un acteur crucial tant pour le maintien des télomères que pour la stabilité du génome dans son ensemble [6-17].

\section{Identification du gène Rtel}

Chez la souris, le gène codant Rtel a été identifié il y a près de 10 ans à la suite de l'observation d'une différence importante dans la taille des 


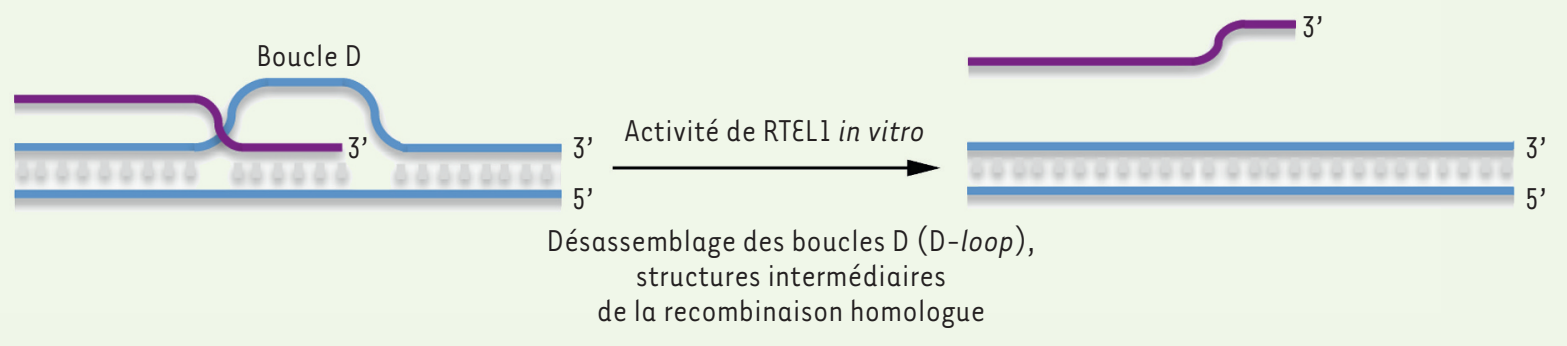

Figure 1. Modèle proposé de l'activité de RTELI in vitro comme anti-recombinase. L'activité hélicase de RTELl, dépendante de l'hydrolyse de l'ATP, permet de désassembler les structures intermédiaires de recombinaison homologue (D-loop).

télomères entre les souris Mus spretus et Mus musculus (communément étudiée en laboratoire) : la taille moyenne des télomères était respectivement comprise entre 5 et 15 kilobases $(\mathrm{kb})$ pour la première et plus de $25 \mathrm{~kb}$ pour la seconde. Un travail remarquable réalisé par clonage positionnel dans le laboratoire de R.J. Hodes a permis d'isoler une région chromosomique de $5 \mathrm{cM}$ (centimorgan) située sur le chromosome 2 qui comporte le trait génétique contrôlant la taille différentielle des télomères dans ces deux souches [18]. Ce n'est que six ans plus tard que l'équipe de P. Lansdorp identifiait dans cette région chromosomique un gène codant une hélicase de l'ADN nommée Rtel [19]. L'invalidation de Rtel par recombinaison homologue dans les cellules souches embryonnaires murines $(\varepsilon S)$ provoque une réduction significative de la taille des télomères, démontrant ainsi le rôle de Rtel dans l'homéostasie de la longueur télomérique [19]. De plus, les cellules ES déficientes en Rtel accumulent de nombreux signes d'instabilité génétique, incluant des fusions télomériques, des cassures chromosomiques et des pertes de télomères lors de leur différenciation [19]. Enfin, l'invalidation du gène Rtel chez la souris (souris knock out, K0) conduit à une létalité embryonnaire vraisemblablement causée par de profonds défauts de développement [19]. Étant donné que les souris KO pour l'activité télomérase sont tout à fait viables et n'ont pas de phénotype particulier avant plusieurs générations, il est vraisemblable que l'effet létal de l'invalidation de Rtell chez la souris est en rapport avec des fonctions bien plus importantes que le maintien de la longueur télomérique. Quoi qu'il en soit, ces données initiales impliquaient Rtel dans les processus régulant la biologie des télomères et la stabilité génomique. Chez l'homme, l'orthologue de Rtel est situé sur le chromosome 20 et correspond au gène RTELI (regulator of telomere elongation helicase 1, OMIM : 608833) initialement nommé NHL (novel helicase like) et associé à des cancers gastriques [19, 20].

\section{RTEL1, un facteur clé de la stabilité génomique}

De très nombreuses hélicases d'ADN jouent un rôle crucial dans la maintenance et la stabilité du génome en coordonnant la recombinaison, la réplication, la réparation, la ségrégation chromosomique, ainsi que la transcription. C'est le cas notamment des enzymes BLM et WRN dont les déficiences sont à l'origine, respectivement, des syndromes de Bloom et de Werner [21]. RTEL1, comme XPD (dont la déficience est responsable du xeroderma pigmentosum), DDXI1 (aussi appelée CHLI ou CHLRl, et dont la déficience est responsable du syndrome Warsaw breakage) et FANC) (dont la déficience est responsable de l'anémie de Fanconi) appartient à une sous-famille d'hélicases d'ADN qui contiennent un domaine fer-soufre ( $\mathrm{Fe}$ S). RTELI requiert l'activité du facteur d'assemblage MMS19 pour le transfert des ions Fe-S, qui lui permet d'être correctement structurée [22-24]. La plupart des informations fonctionnelles dont nous disposons pour RTELl proviennent d'études réalisées chez le nématode Caenorhabditis elegans et la souris. II a en particulier été démontré que RTELl participait à la régulation du mécanisme de recombinaison homologue, un processus clé, non seulement dans la réparation des cassures double brin de l'ADN, mais également dans la dynamique et le redémarrage des fourches de réplication arrêtées [7]. Les vers C. elegans déficients pour rtel-1 présentent une augmentation des intermédiaires de recombinaison homologue, une hypersensibilité à des agents génotoxiques, tels que la camptothécine, ainsi qu'une augmentation de la recombinaison méiotique $[7,14]$. De plus, des lignées cellulaires humaines dans lesquelles le taux de RTELl a été diminué par interférence ARN ont un taux de recombinaison homologue augmenté et une sensibilité accrue à la mitomycine $C(M M C)$, un agent créant des ponts d'ADN interbrins [7]. Des expériences in vitro utilisant la protéine RTEL] humaine purifiée ont suggéré que cette dernière jouait un rôle suppresseur de la recombinaison homologue ou «anti-recombinase homologue » en dissociant les structures intermédiaires de la recombinaison homologue nommées boucles D (Figure 1) [7]. II a ensuite été démontré que cette activité anti-recombinase jouait un rôle dans le contrôle de la fréquence des recombinaisons méiotiques chez C. elegans [14], puisque le taux de recombinaison méiotique (crossing over) est anormalement élevé chez les nématodes déficients en rtel-1 [14]. Dans cette même étude, des expériences in vitro avec une protéine RTELl humaine 

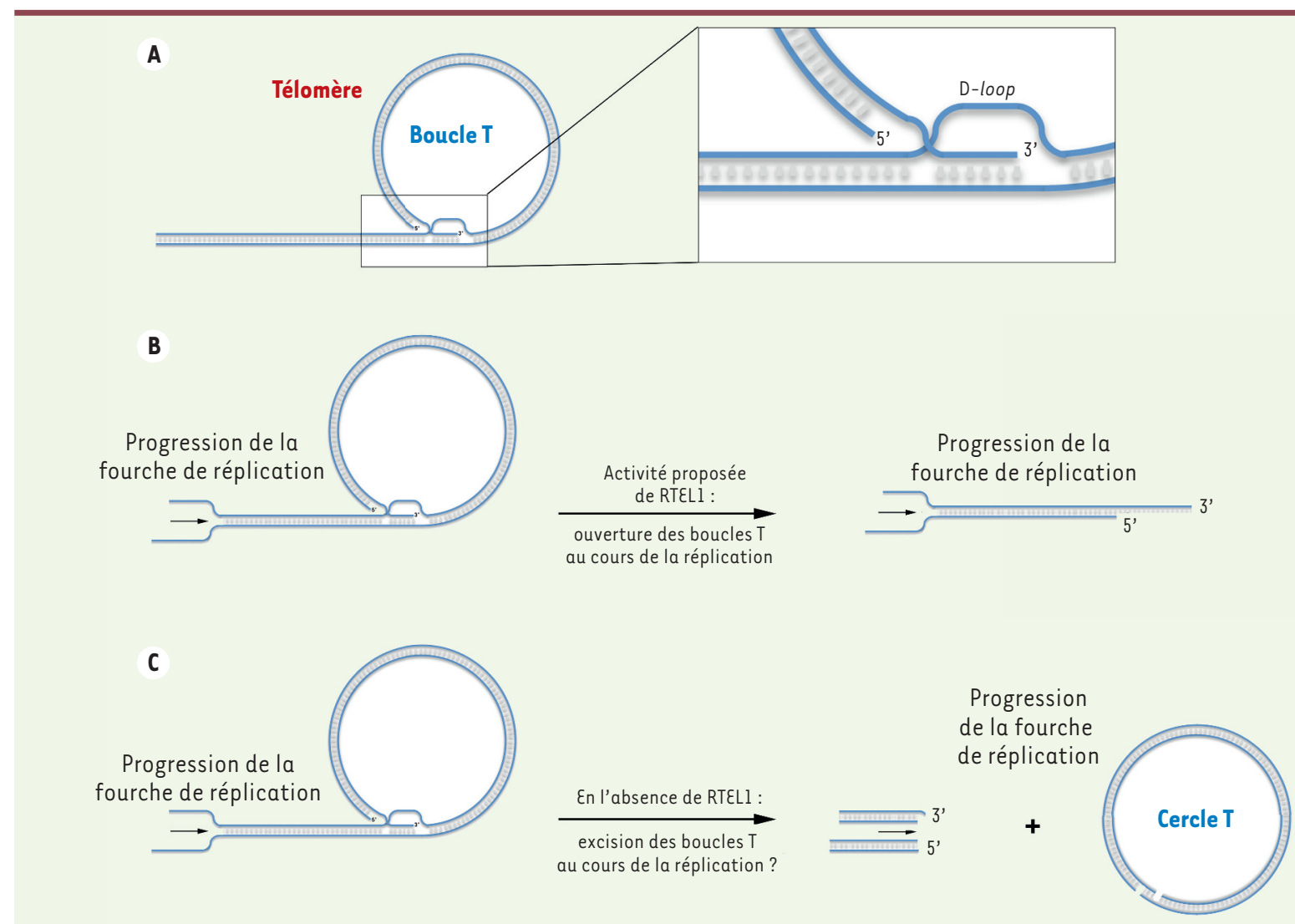

Figure 2. Modèle proposé de l'activité de RTELI au cours de la réplication des télomères. A. Les télomères peuvent adopter une structure en boucle (T-loop) formée par l'invasion du surplomb télomérique 3' dans la séquence télomérique double brin. Cette structure particulière forme une boucle $D$ (voir zoom). B. Au cours de la réplication des télomères, RTELl pourrait dissocier la boucle $D$ télomérique et, ainsi, permettre une réplication complète des télomères. $C$. En absence de RTELl, au cours de la réplication, les boucles T ne pourraient être désassemblées et génèreraient, après excision, la formation de cercles $T$.

purifiée ont permis de préciser que la dissociation de la boucle $D$ relayée par RTELl agit préférentiellement sur les invasions des extrémités 3' de l'ADN (Figure 1), et nécessite la présence de RPA (replication protein $A$ ), une protéine interagissant spécifiquement avec l'ADN simple brin [14].

Une première étude réalisée dans des fibroblastes embryonnaires de souris (MEF, murine embryonic fibroblasts) dans lesquels l'expression de Rtell avait été réduite par interférence à ARN montrait une augmentation de la fragilité télomérique (caractérisée par de multiples signaux télomériques sur une même chromatide). Cela suggèrait un rôle de Rtell dans la réplication correcte des télomères [10].

Plus récemment, l'étude du phénotype de MEF dont le gène Rtell a été invalidé de façon conditionnelle ( $K O$ conditionnel) a permis de préciser les conséquences au niveau des télomères de la suppression de Rtell chez la souris [12]. On observe dans les fibroblastes embryonnaires murins déficients en Rtell une augmentation des cassures chromosomiques, des pertes télomériques sur une ou deux chromatides sœurs, ainsi que des signes de fragilité télomérique [5, 12]. Dans cette étude, les auteurs proposent qu'une des conséquences d'un défaut en Rtell soit l'excision inappropriée des télomères sous forme de cercles (cercle T, T-circle) au cours de la réplication. En effet, les télomères peuvent adopter une structure en boucle (boucle T, T-loop) via l'invasion de l'extrémité 3' simple brin télomérique dans la séquence double brin télomérique (Figure 2) [5, 12, 25]. Par conséquent, cette structure, similaire à la D-loop, pourrait être dissociée par Rtell afin de permettre une réplication complète des télomères. Cette proposition semble être confortée par l'observation d'une augmentation des cercles $T$ au cours de la réplication des cellules déficientes en Rtell [12] (Figure 2). Dans ce contexte, l'excision de la boucle $T$ semble principalement impliquer le complexe nucléase SLX4 [12, 26]. Par ailleurs, Vannier et al. suggèrent qu'un autre rôle de Rtell serait de dissocier les G-quadruplexes (des structures complexes d'ADN présentes aux télomères qui perturbent la réplication) et, ainsi, de protéger les télomères de lésions délétères et d'éviter l'expression d'un phénotype de télomères fragiles $[10,12]$. Une autre étude réalisée par Uringa et al. dans des cellules souches embryonnaires murines 


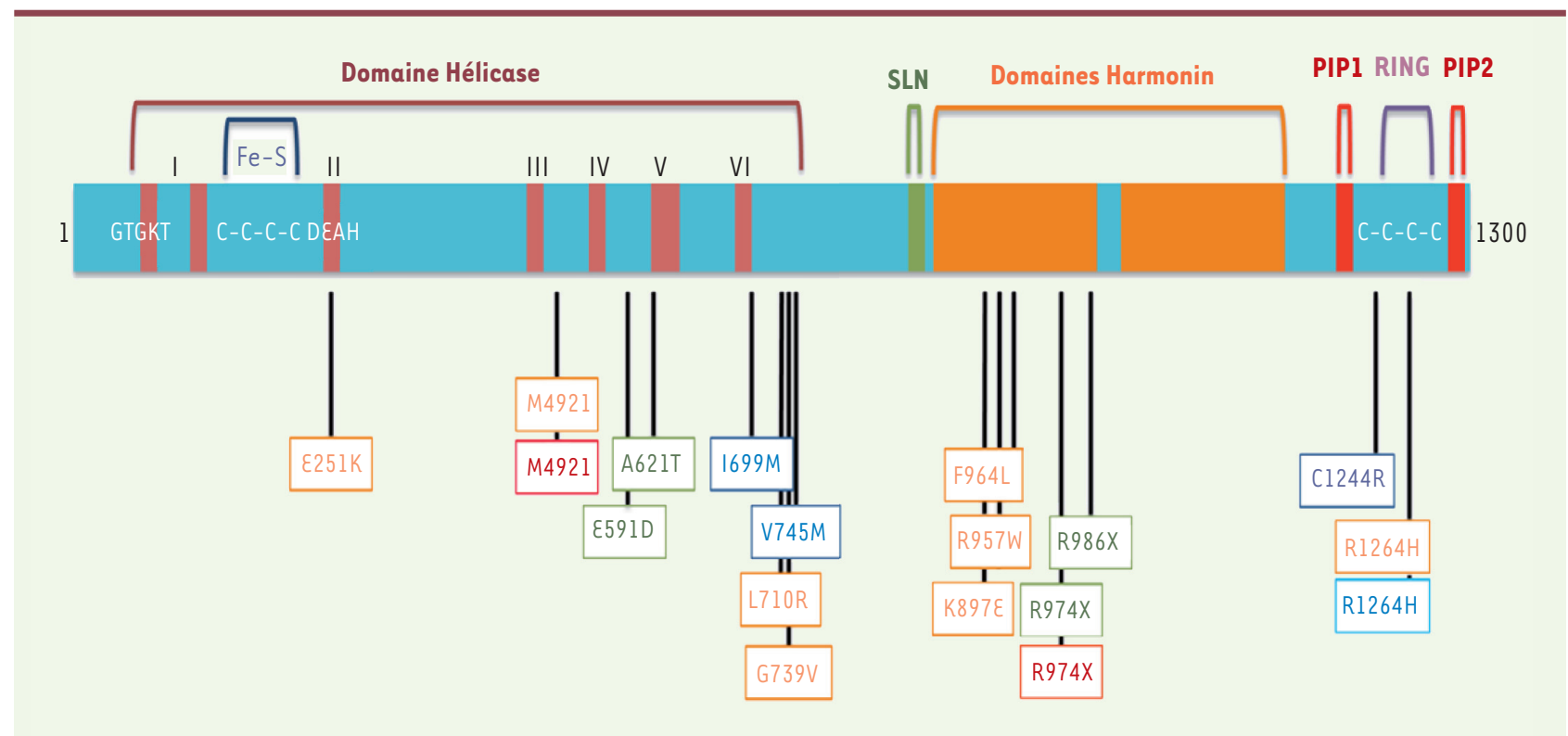

Figure 3. Schéma de la forme longue de RTEL1 humaine (1 300 acides aminés). Les différents domaines, ainsi que les mutations identifiées chez les patients atteints de dyskératose congénitale et du syndrome Hoyeraal-Hreidarsson sont représentés [6, 8, 9, 13, 15]. SLN : signal de localisation nucléaire ; PIP : PCNA interacting protein box.

invalidées pour Rtell a confirmé le rôle important qu'exerce Rtell pour répliquer correctement les télomères [11]. Cependant, sa participation dans l'ouverture des boucles T pour éviter les pertes télomériques n'a pas été confirmée. En effet, contrairement aux résultats obtenus dans les MEF déficients en Rtell [12], l'augmentation des cercles T n'a pas été observée dans les cellules \&S déficientes en Rtell [11]. Cette différence pourrait provenir du type de cellules étudiées (notamment la possibilité d'une adaptation à l'absence de Rtell dans des cellules indifférenciées), ainsi que des méthodes différentes utilisées pour détecter les cercles T. Enfin, l'étude menée dans les cellules ES déficientes en Rtell par Uringa et al. démontre l'implication de Rtell dans la réparation des ponts interbrins de l'ADN générés par la MMC et, dans une moindre mesure, des lésions produites par les radiations ionisantes et les ultraviolets [11].

\section{Déficience en RTELl chez l'homme}

Chez l'homme, un défaut congénital de protection des télomères et/ou de maintenance de leur longueur conduit à la dyskératose congénitale (DC), une maladie génétique rare dont les signes cliniques sont l'aplasie médullaire, un vieillissement accéléré, et une prédisposition aux cancers [5]. Le syndrome de Hoyeraal-Hreidarsson (HH), une variante rare et sévère de dyskératose congénitale, associe au moins quatre des six caractéristiques suivantes : insuffisance médullaire précoce, retard de croissance intra-utérin, défauts du développement, microcéphalie, hypoplasie cérébelleuse et immunodéficience [5]. Des mutations dans plusieurs gènes codant des facteurs impliqués dans la régulation de la taille et/ou de la protection des télomères ont été identifiées dans ces pathologies : DKCl (dyskerin), TERC (telomerase RNA component), TERT (telomerase reverse transcriptase), TIN2 (TRF1-interacting nuclear protein 2), NOP10, NHP2 (non-histone protein 2), TCABI (telomerase cajal body protein 1), Apollo, et CTCl (CST complex protein 1) [5]. Néanmoins, environ $40 \%$ des DC/HH n'ont encore aucune cause moléculaire identifiée. En 2013, quatre études indépendantes ont caractérisé des patients atteints de $\mathrm{DC} / \mathrm{HH}$ et porteurs de mutations du gène RTELI (Figure 3) [6, 8, 9, 13, 15]. Au total, ces différentes explorations permettent de décrire à ce jour 14 patients atteints de $\mathrm{DC} / \mathrm{HH}$ porteurs de mutation dans le gène RTELI. Dans la plupart des cas, la transmission génétique est autosomique récessive (les patients portent des mutations homozygotes ou hétérozygotes composites). Chez deux patients d'une même famille, une seule mutation hétérozygote dans le gène RTELI a été identifiée, suggérant une transmission autosomique dominante avec anticipation de la maladie (la mère porte également la mutation sans présenter de signe clinique) [6]. Cependant, on ne peut exclure chez ces patients l'existence d'une altération du gène RTELI en dehors des exons (et éventuellement conduisant à des défauts d'expression ou d'épissage) qui n'aurait pas été détectée par le séquençage d'exome. Puisqu'un défaut complet en Rtell est létal chez la souris [19], il est très probable que les mutations identifiées chez les patients soient hypomorphes. Tous les patients déficients en RTELl décrits à ce jour ont des télomères anormalement courts pour leurs âges, confirmant le rôle de RTELI dans la régulation de la taille des télomères chez l'homme 


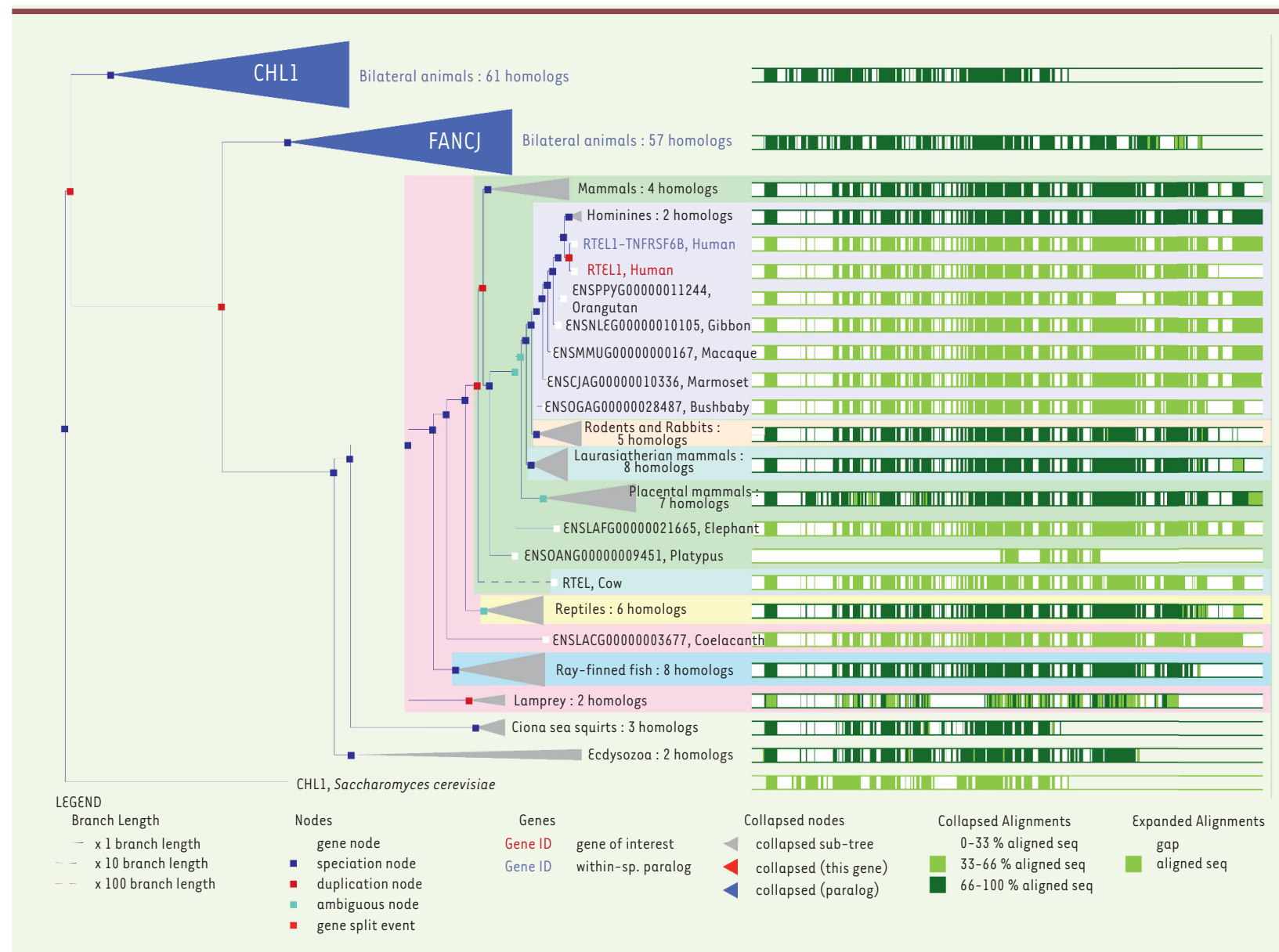

Figure 4. Arbre phylogénétique du gène RTELl et de ses paralogues CHL1 et FANCJ. On remarque la présence des séquences en position carboxyterminale propre aux vertébrés. Arbre phylogénétique réalisé dans Ensembl (http://www.ensembl.org/index.html).

$[6,8,9,13,15]$. Par ailleurs, une étude phénotypique réalisée dans les cellules de trois patients déficients en RTELl a mis en évidence la présence inhabituelle de ponts d'anaphase (signe d'instabilité génomique), un nombre de foyers 53BPl spontanés (marqueurs de cassures de l'ADN) anormalement élevé, ainsi que des aberrations télomériques (pertes sèches, délétions terminales et échanges de séquences télomériques entre deux chromatides sœurs), sans signe d'instabilité chromosomique générale [9]. Curieusement, et contrairement à ce qui a été observé dans des lignées humaines déplétées en RTELl par interférence à ARN [7], Walne et al. n'ont pas noté d'altération de la recombinaison homologue dans les cellules de patients déficients en RTELI (conclusions basées sur l'absence d'augmentation de cassure chromosomique après MMC ou diépoxybutane [DEB]) [13]. Par ailleurs, la même étude relate une légère augmentation du nombre de cercles T dans les cellules de ces patients. Cependant, deux autres études indépendantes n'ont pu confirmer la présence (ou l'augmentation) de cercles $T$ dans différents types de cellules de plusieurs patients déficients en RTELI (T. Le Guen et P. Revy, données non publiées, et [8]). Ces résultats divergents peuvent résulter de la nature différente des mutations portées par ces patients et/ou des méthodes utilisées pour détecter les cercles T. L'identification de mutations de RTELl chez des patients atteints de DC/HH démontre le rôle essentiel joué par RTELl chez l'homme pour garantir la stabilité du génome et le maintien des télomères. Il est envisageable que des mutations moins délétères de RTELl ou des dérégulations de son expression puissent être associées au développement de cancers. Cette hypothèse est renforcée par l'observation que des souris transgéniques pour Rtell développent des tumeurs hépatiques [27], et que des souris portant une mutation hypomorphe de Rtell à l'état homozygote présentent une susceptibilité accrue au développement de cancers (lymphomes, sarcomes, tératomes et médulloblastomes) lorsqu'elles sont croisées avec les souris déficientes en $\mathrm{p} 53$ (utilisées comme accélérateur de tumeur) [17]. De plus, plusieurs études ont identifié des polymorphismes dans des régions non codantes du gène RTELl, associés à des susceptibilités accrues à développer des cancers, dont des gliomes de haut grade [28-30]. 


\section{Fonctions de RTELl : de nombreuses questions demeurent}

Bien que les différents travaux décrits précédemment aient établi une implication de RTELI dans la réplication, la régulation de la taille et la stabilité des télomères, ainsi que dans la réparation de certaines lésions de l'ADN, de nombreuses zones d'ombre demeurent quant à ses modes d'action. Outre son domaine hélicase, l'analyse structurale de RTELl donne des indices sur ses éventuels modes d'action et ses partenaires (Figure 3). Ainsi, deux motifs PIP (proliferating cell nuclear antigen $[P C N A]$ interacting protein) permettant l'interaction avec PCNA, un facteur impliqué dans la réplication et la réparation de I'ADN, le remodelage chromatinien et la régulation du cycle cellulaire [31], sont présents dans la région carboxy-terminale de la protéine. L'interaction de RTELl avec PCNA via le premier domaine PIP a été rapportée très récemment chez la souris, et semble être importante pour permettre une progression optimale des fourches de réplication et éviter les effets délétères du stress réplicatif [17]. Chez l'homme, deux isoformes de RTELl sont principalement exprimées. Ces isoformes, générées par épissage alternatif, diffèrent par leur extrémité carboxy-terminale [9]. De façon très intéressante, la forme longue de RTELl possède à son extrémité carboxy-terminale un domaine RING très similaire à celui qui est présent dans $B M I-l$, une protéine à activité d'ubiquitination (ubiquitinase ou ubiquitine ligase) [9]. Par conséquent, RTELl pourrait avoir, via son domaine RING, une activité d'ubiquitination, interagir avec des ubiquitinases de type $\varepsilon 3$ ou bien permettre des homo- ou hetérodimérisations [9]. Étant donné que ce domaine est encadré par deux motifs PIP, une possibilité intéressante serait que ces activités d'ubiquitination soient dépendantes, ou du moins coordonnées par, une interaction avec PCNA, par exemple pour la réparation dépendante de la réplication. II est à noter que les domaines présents dans la partie carboxy-terminale de RTELl sont apparus au cours de l'évolution chez les vertébrés (Figure 4). De plus, deux domaines Harmonin [32] ont été identifiés dans RTELI [16]. Ces domaines, localisés en aval du signal de localisation nucléaire, pourraient permettre l'interaction avec des partenaires qui restent à identifier. Enfin, bien que le rôle initial attribué à RTELI ait été la régulation de la taille des télomères [19], son mode d'action dans ce processus reste encore largement méconnu. De même, les pertes télomériques observées dans les cellules déficientes en RTELl ne peuvent sans doute pas rendre compte à elles seules de la réduction globale de la taille des télomères dans ce contexte. Par conséquent, il est probable que RTELl participe, directement ou indirectement, à la régulation de l'activité de la télomérase. Des analyses précises des divers domaines de RTELl et de ses partenaires sont nécessaires pour mieux caractériser les multiples fonctions qu'exerce cette protéine pour assurer la stabilité du génome, en particulier chez l'homme. $\diamond$

\section{SUMMARY}

RTEL1 (regulator of telomere elongation helicase 1), a DNA helicase essential for genome stability

RTELI (regulator of telomere length helicase 1) is a DNA helicase that has been identified more than 10 years ago. Many works since, mainly in the nematode Caenorhabditis elegans and the mouse, have highlighted its role in chromosomal stability, maintenance of telomere length, and DNA repair. Recently, four laboratories have characterized RTELl mutations in patients with dyskeratosis congenita (DC) and Hoyeraal-Hreidarsson (HH) syndrome, a rare and severe variant of DC. We here summarize the current knowledge on RTELl and discuss the possible other functions that RTELI could play. $\diamond$

\section{LIENS D'INTÉRÊT}

Les auteurs déclarent n'avoir aucun lien d'intérêt concernant les données publiées dans cet article.

\section{RÉFÉRENCES}

1. Gilson E, Geli V. How telomeres are replicated. Nat Rev Mol Cell Biol 2007 ; 8 : 825-38.

2. Von Zglinicki T. Oxidative stress shortens telomeres. Trends Biochem Sci $2002: 27: 339-44$.

3. Londoño-Vallejo JA. Un Nobel centenaire célèbre télomères et télomérase. Med Sci (Paris) 2009 ; 25 : 973-6.

4. De Lange T. How shelterin solves the telomere end-protection problem. Cold Spring Harb Symp Quant Biol 2009 ; 75 : 167-77.

5. Touzot F, Le Guen T, de Villartay JP, Revy P. Nouvelles formes de dyskératoses congénitales - Le dysfonctionnement des télomères n'est pas systématiquement associé à une réduction de leur taille. Med Sci (Paris) $2012 ; 28: 618-24$.

6. Ballew BJ, Yeager M, Jacobs K, et al. Germline mutations of regulator of telomere elongation helicase 1, RTELl, in Dyskeratosis congenita. Hum Genet 2013 ; 132 : 473-80.

7. Barber LJ, Youds JL, Ward JD, et al. RTELl maintains genomic stability by suppressing homologous recombination. Cell 2008 ; 135 : 261-71.

8. Den, Z, Glousker G, Molczan A, et al. Inherited mutations in the helicase RTELl cause telomere dysfunction and Hoyeraal-Hreidarsson syndrome. Proc Natl Acad Sci USA 2013 (sous presse).

9. Le Guen T, Jullien L, Touzot F, et al. Human RTELl deficiency causes Hoyeraal-Hreidarsson syndrome with short telomeres and genome instability. Hum Mol Genet 2013 ; 22 ; 3239-49.

10. Sfeir A, Kosiyatrakul ST, Hockemeyer D, et al. Mammalian telomeres resemble fragile sites and require TRFl for efficient replication. Cell 2009 ; $138: 90-103$

11. Uringa $\varepsilon$ J, Lisaingo K, Pickett HA, et al. RTELl contributes to DNA replication and repair and telomere maintenance. Mol Biol Cell $2012 ; 23: 2782-92$.

12. Vannier JB, Pavicic-Kaltenbrunner V, Petalcorin MI, et al. RTELl dismantles T loops and counteracts telomeric G4-DNA to maintain telomere integrity. Cell $2012 ; 149: 795-806$.

13. Walne AJ, Vulliamy T, Kirwan M, et al. Constitutional mutations in RTELl cause severe dyskeratosis congenita. Am J Hum Genet 2013 ; 92 : 448-53.

14. Youds JL, Mets DG, Mcllwraith MJ, et al. RTEL-1 enforces meiotic crossover interference and homeostasis. Science 2010 ; 327 : 1254-8.

15. Ballew BJ, Joseph V, De Z, et al. A recessive founder mutation in regulator of telomere elongation helicase l, RTELl, underlies severe immunodeficiency and features of Hoyeraal Hreidarsson syndrome. PLoS Genet 2013 ; 9 : e1003695.

16. Faure $G$, Revy P, Schertzer M, et al. The C-terminal extension of human RTELl, mutated in Hoyeraal-Hreidarsson syndrome, contains Harmonin- $\mathrm{N}$ like domains. Proteins 2013 ; doi:10.1002/prot.24438.

17. Vannier JB, Sandhu S, Petalcorin MI, et al. RTELl is a replisome-associated helicase that promotes telomere and genome-wide replication. Science $2013 ; 342: 239-42$.

18. Zhu L, Hathcock KS, Hande P, et al. Telomere length regulation in mice is linked to a novel chromosome locus. Proc Natl Acad Sci USA 1998 ; 95 : 8648-53.

19. Ding H, Schertzer $M$, Wu X, et al. Regulation of murine telomere length by Rtel: an essential gene encoding a helicase-like protein. Cell $2004 ; 117$ : 873-86.

20. Bai C, Connolly B, Metzker ML, et al. Overexpression of M68/DcR3 in human gastrointestinal tract tumors independent of gene amplification and its location in a four-gene cluster. Proc Natl Acad Sci USA 2000 ; 97 : 1230-5. 


\section{RÉFÉRENCES}

21. Bohr VA. Rising from the Rec $\rho$-age: the role of human Rec $\varrho$ helicases in genome maintenance. Trends Biochem Sci $2008 ; 33: 609-20$.

22. Gari K, Leon Ortiz AM, Borel V, et al. MMS19 links cytoplasmic iron-sulfur cluster assembly to DNA metabolism. Science 2012 ; $337: 243-5$.

23. Stehling 0, Vashisht AA, Mascarenhas J, et al. MMS19 assembles iron-sulfur proteins required for DNA metabolism and genomic integrity. Science $2012 ; 337$ : 195-9.

24. Uringa $\varepsilon$ J, Youds JL, Lisaingo K, et al. RTELl: an essential helicase for telomere maintenance and the regulation of homologous recombination. Nucleic Acids Res 2011 ; 39 : 1647-55.

25. Griffith JD, Comeau L, Rosenfield S, et al. Mammalian telomeres end in a large duplex loop. Cell $1999 ; 97: 503-14$.

26. Fekairi S, Scaglione S, Chahwan C, et al. Human SLX4 is a Holliday junction resolvase subunit that binds multiple DNA repair/recombination endonucleases. Cell $2009: 138: 78-89$.

27. Wu X, Sandhu S, Nabi Z, Ding H. Generation of a mouse model for studying the role of upregulated RTELl activity in tumorigenesis. Transgenic Res $2012 ; 21$ : 1109-15.

28. Egan KM, Thompson RC, Nabors LB, et al. Cancer susceptibility variants and the risk of adult glioma in a US case-control study. J Neurooncol $2011 ; 104: 535-42$.
29. Liu Y, S Shete, CJ Etzel, et al. Polymorphisms of LIG4, BTBD2, HMGA2, and RTELl genes involved in the double-strand break repair pathway predict glioblastoma survival. J Clin Oncol $2010 ; 28: 2467-74$.

30. Wrensch M, Jenkins RB, Chang JS, et al. Variants in the CDKN2B and RTELI regions are associated with high-grade glioma susceptibility. Nat Genet $2009 ; 41: 905-8$.

31. Maga G, Hubscher U. Proliferating cell nuclear antigen (PCNA): a dancer with many partners. J Cell Sci 2003 ; 116 : 3051-60.

32. Verpy $\varepsilon$, M Leibovici, I Zwaenepoel, et al. A defect in harmonin, a PDZ domain-containing protein expressed in the inner ear sensory hair cells, underlies Usher syndrome type 1C. Nat Genet $2000 ; 26: 51-5$.

\section{Collection SCIENCE ET BIOMÉDECINE}

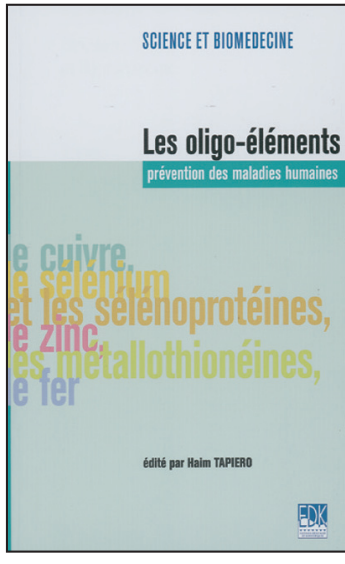

ISBN : 2-84254-107-3 64 pages

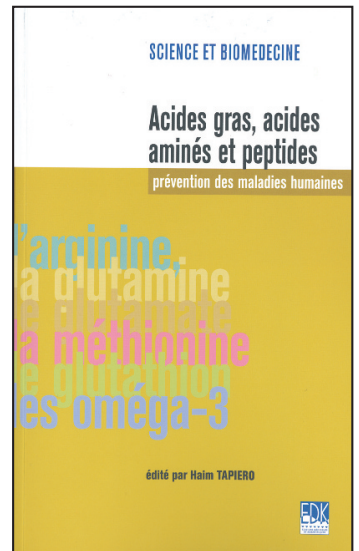

ISBN : 2-84254-108-1 80 pages
TIRÉS À PART

P. Revy

\section{Bon de commande}

À retourner à EDK, 25, rue Daviel - 75013 Paris

Tél. : 0158101905 - Fax : 0143293262 - E-mail : edk@edk.fr

NOM : Prénom:

Adresse :

Code postal : Ville :

Pays :

Fonction :

Je souhaite recevoir l'ouvrage Les oligo-éléments : $10 €+3 €$ de port $=\mathbf{1 3} €$ TTC

Je souhaite recevoir l'ouvrage Acides gras, acides aminés et peptides : $12 €+3 €$ de port $=15 €$ TTC

Je souhaite recevoir l'ouvrage Stress oxydatif et alicaments : $14 €+3 €$ de port $=\mathbf{1 7} €$ TTC

$\square$ Par chèque, à l'ordre de $\mathbf{E} \mathbf{D}$ K
$\square$ Par carte bancaire :
$\square$ Visa $\square$ Eurocard/Mastercard

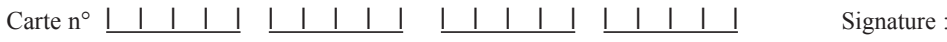

Date d'expiration: $\quad|\quad| \quad|\quad|$

$\mathrm{N}^{\circ}$ de contrôle au dos de la carte : $\quad$ I $\quad$ | 1 